\title{
Tissue-Specific Sparse Deconvolution for Low-Dose CT Perfusion
}

\author{
Ruogu Fang ${ }^{1}$, Tsuhan Chen $^{1}$, and Pina C. Sanelli ${ }^{2,3}$ \\ 1 Department of Electrical and Computer Engineering, Cornell University, Ithaca, NY, USA \\ ${ }^{2}$ Department of Radiology, Weill Cornell Medical College, New York, NY, USA \\ ${ }^{3}$ Department of Public Health, Weill Cornell Medical College, New York, NY, USA
}

\begin{abstract}
Sparse perfusion deconvolution has been recently proposed to effectively improve the image quality and diagnostic accuracy of low-dose perfusion CT by extracting the complementary information from the high-dose perfusion maps to restore the low-dose using a joint spatio-temporal model. However the low-contrast tissue classes where infarct core and ischemic penumbra usually occur in cerebral perfusion CT tend to be over-smoothed, leading to loss of essential biomarkers. In this paper, we extend this line of work by introducing tissuespecific sparse deconvolution to preserve the subtle perfusion information in the low-contrast tissue classes by learning tissue-specific dictionaries for each tissue class, and restore the low-dose perfusion maps by joining the tissue segments reconstructed from the corresponding dictionaries. Extensive validation on clinical datasets of patients with cerebrovascular disease demonstrates the superior performance of our proposed method with the advantage of better differentiation between abnormal and normal tissue in these patients.
\end{abstract}

\section{Introduction}

Computed tomography perfusion (CTP) [1] has been more commonly used in patients with cerebrovascular diseases to characterize tissue perfusion. Specifically, in acute stroke patients, detection of ischemic regions has been a main focus in the literature. The associated excessive radiation exposure of CTP has aroused great concern due to overdosage leading to biological effects including hair loss, skin burn and increased cancer risk [2]. Even currently recommended CTP scanning parameters still contribute to increased lifetime cancer risk. Sparse perfusion deconvolution (SPD) [3] [4] is a recently proposed method for low-dose CTP deconvolution. Different from previous methods [5][6], whose image prior has been based on some simplifying assumptions, SPD is a data-driven method that restores the input low-dose perfusion map using a spatiotemporal model. The model is regularized by a sparse combination of atoms from a global dictionary learned from the high-dose perfusion maps. In this way, spatial priors are incorporated on-the-fly. SPD is able to remove the noise and can preserve the vascular structure and contrast in low-dose perfusion maps.

Theoretically, a global dictionary is able to capture sufficient image information for different tissue classes, given abundant training data from each class. Learned global dictionaries have been applied to various domains including image super-resolution [7] and deformable shape modeling [8]. However empirically the optimization procedure

K. Mori et al. (Eds.): MICCAI 2013, Part I, LNCS 8149, pp. 114-121, 2013.

(c) Springer-Verlag Berlin Heidelberg 2013 
which minimizes the overall reconstruction error, tends to favor high-contrast patches to the low-contrast ones in both the learning and reconstruction procedures. For medical images, the subtle variations and changes embedded in the low-contrast tissue classes such as white matter can be crucial for disease detection and diagnosis [9].

In this paper, we propose a tissue-specific sparse deconvolution method to address the limitations above. Our method starts from segmenting the brain into different tissue classes. A modified version of automated model-based tissue classification [10] is employed to segment the brain tissue classes. Then tissue-specific dictionaries are learned from the training segments of each class. Finally we use weighted sparse deconvolution method to restore each tissue class and stitch them together. The extensive experiments demonstrate the superior performance of our method. It is important to note that all the preprocessing methods to denoise the dynamic CT data can be complimented with our proposed deconvolution algorithm to achieve better performance.

Our main contribution is two-fold: (1) Tissue-specific dictionaries for each tissue class are employed in place of the global dictionary to capture the low-contrast tissue class and delicate structural details. (2) Weighted sparse deconvolution based on the probability of the tissue classification is proposed for a unified reconstruction of the low-dose perfusion maps. In vivo brain acute stroke and aneurysmal SAH patients data, we demonstrate the superiority of our proposed method in CBF estimation that leads to better separation between normal and ischemic tissue.

\section{Tissue-Specific Approach to Sparse Deconvolution}

\subsection{Tissue Classification}

We first classify the voxels in the dynamic brain CTP data into four tissue classes: vessel, gray matter (GM), white matter (WM) and cerebrospinal fluid (CSF). Since computational efficiency is very important in our framework for real-time clinical diagnosis, we choose a simple yet effective segmentation approach by adapting a tissue classification algorithm for MRI [10]. We first compute the median value for each voxel along the temporal axis since different tissue classes have different contrast perfusion characteristics. Expectation-maximization segmentation is employed on the median map to obtain probability maps of GM, WM and CSF, while contexture information is incorporated by a Markov Random Field (MRF). The reason for choosing median map as a robust measurement of the tissue contrast in CTP is because of its higher tissue contrast compared to other statistics in our experiments. Vessel is segmented by thresholding the original CBF value. The vessel voxels in other tissue probability maps are set to zero to guarantee mutually occlusive segmentations. Tissue probability maps on a representative dataset are shown in Fig. 1. The following reconstruction does not heavily depend on the segmentation accuracy, since each tissue dictionary is learned from over 10,000 patches and represents dominant patterns in the training patches.

\subsection{Tissue-Specific Dictionary Learning}

Based on the tissue classification from the previous section, we obtain $M$ sets $S_{m}$ of training patches, $m=V$ essel, $G M, W M, C S F$, by classifying a patch $y$ from a 


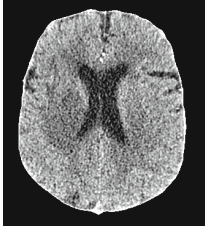

(a)

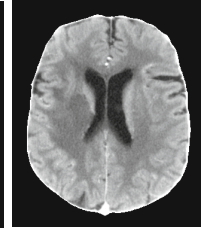

(b)

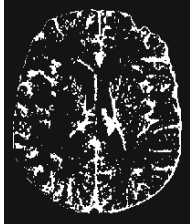

(c)

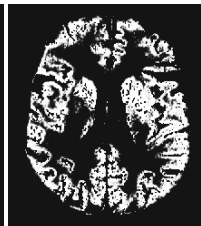

(d)

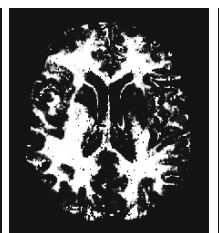

(e)

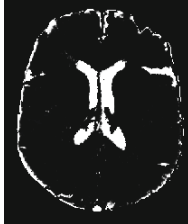

(f)

Fig. 1. Brain tissue classification by the automatic algorithm on the median map. (a) A slice in the enhanced CTP data (b) Median map. Probability maps of (c) Vessel (d) Gray matter (e) White matter (f) CSF.

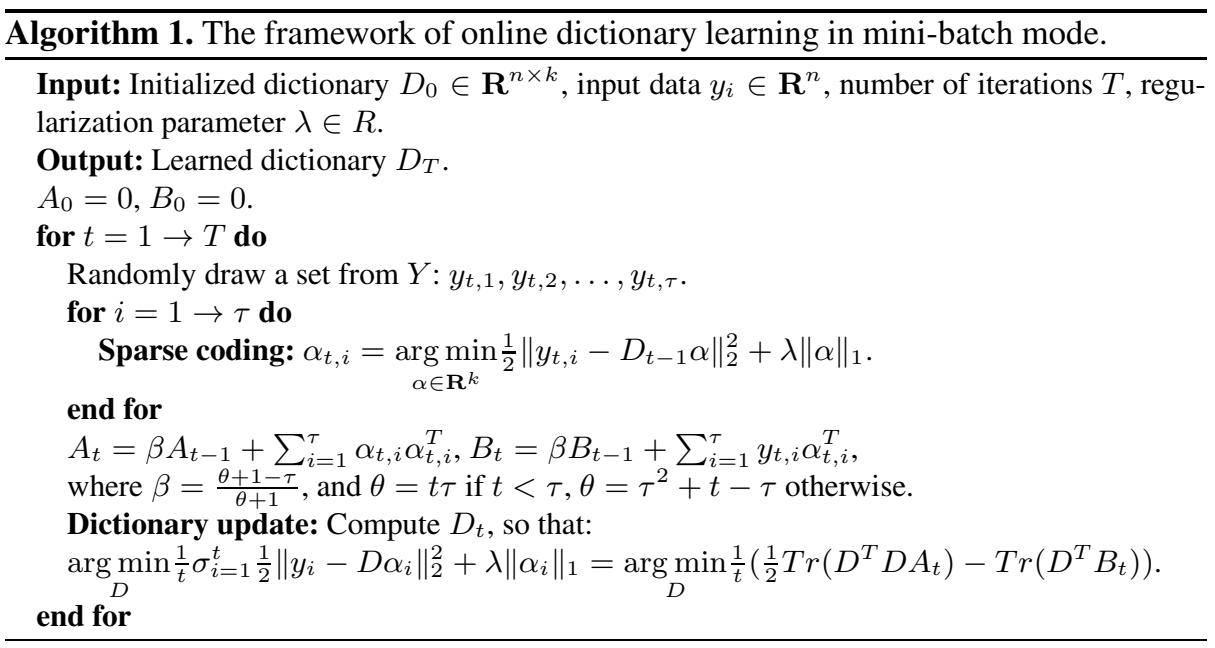

training image to class $i$ if more than $50 \%$ of voxels in the patch $y$ belongs to class $i$. Voxels from other classes are then removed from the patch.

To learn the tissue-specific dictionary $\mathbf{D}^{m}, m=V$ essel, $G M, W M, C S F$, we use the recently developed online learning algorithm [11] which is able to update the dictionary with every batch of new training samples and avoids the time-consuming reconstruction of the entire dictionary when new samples come. Given a set of high-dose CBF patches $Y^{m}=\left\{y_{i}^{m}\right\}_{i=1}^{N}$ for a specific tissue type $m$, each as a column vector of size N. $\alpha_{i}$ in $R^{K}$ is a sparse vector to make $\mathbf{D} \alpha_{i}$ an approximation to $y_{i}^{m}$ with certain error tolerance. $A^{m}=\left[\alpha_{1}, \ldots, \alpha_{N}\right]$. We seek the dictionary $\mathbf{D}^{m}$ in $\mathbf{R}^{N \times K}$ in that minimizes

$$
\min _{\mathbf{D}^{m}, \mathbf{A}^{m}} \sum_{i=1}^{N}\left\|y_{i}^{m}-\mathbf{D}^{m} \alpha_{i}\right\|_{2}+\mu_{2}\left\|\alpha_{i}\right\|_{1}
$$

The framework of online dictionary learning is depicted in Algorithm 1 . The dictionary is updated efficiently using block-coordinate descent based on stochastic approximation. Because it only exploits a small batch of newly coming data in the dictionary update step, it is therefore much faster than K-SVD or other off-line learning algorithms. 
Since computational efficiency is very important in our framework, we can efficiently update the tissue-specific dictionaries with newly coming data using online learning. Moreover online learning also does not require the loading of all data the same time, which is unfeasible in clinical practice, and results in less memory cost.

\subsection{Weighted Sparse Deconvolution}

Let's assume dynamic CTP data $\mathbf{C}$ in $\mathbf{R}^{N \times T}$ composed of $N$ tissue enhancement curves (TEC) at voxels of interest (VOI) $[x, y, z]^{T}$ and $T$ time points. The residue impulse function (RIF) is represented by $\mathbf{R}$ in $\mathbf{R}^{N \times T}$, indicating the delaying of the remaining contrast tracer in the VOI. $f$ in $\mathbf{R}^{N}$ is the CBF map to be estimated and $\mathbf{D}$ is the learned dictionary. The deconvolution step in SPD algorithm computes the CBF map of lowdose CTP data using both temporal convolution model and tissue-specific dictionarybased spatial regularization by solving:

$$
J=\mu_{1}\left\|\mathbf{C}^{m}-\mathbf{C}_{\mathbf{a}} \mathbf{R}^{m}\right\|_{2}^{2}+\left\|f^{m}-\mathbf{D}^{m} \alpha\right\|_{2}^{2}+\mu_{2}\|\alpha\|_{1}
$$

where $\mathbf{C}^{m}, \mathbf{R}^{m}, \mathbf{D}^{m}$ and $f^{m}$ are the corresponding TEC, RIF, dictionary and CBF for tissue class $m$ for a patch of size $N \times N$. The final global CBF parametric map is generated by averaging the areas of neighboring patches with overlap of one pixel.

Eq. (2) is solved by an EM style algorithm with iterative employment of two processes: 1) sparse coding process which minimizes with respect to $\alpha$ with $f$ fixed, 2) quadratic solver which efficiently minimizes this simplified linear inverse problem, as in [4]. Two procedures are iteratively employed to obtain $f^{m}$ and $\alpha^{m}$ for each tissue type. Proper initialization in Eq. (2) with the output of cTSVD poses the optimization at a good start point and is supposed to mitigate local minima. We also observe our results are quite stable with respect to the training dataset.

The probability map of each tissue class is obtained by employing the model-based segmentation algorithm [10] on the median map of the low-dose CTP data. For every tissue class $m$, a tissue-specific patch $\hat{f}_{i}^{m}$ is reconstructed using the corresponding tissue-specific dictionary of tissue class $m$. The patch $\hat{f}_{i}^{m}$ is then weighted by the probability map of class $m$ for patch $f_{i}$ and all probability-weighted tissue-specific patches are summed together to obtain the final reconstruction.

Using tissue-specific dictionaries to enhance low-dose CTP maps, SPD obtains three additional advantages: 1) Segmentation information is incorporated into the dictionary learning and reconstruction. 2) Each tissue type has sufficient atoms in the tissuespecific dictionary to reconstruct. 3) Tissue specific parameter settings can be employed according to the spatial smoothness of each tissue class.

\section{Experiments}

To evaluate the performance of the proposed tissue-specific sparse deconvolution (TSSPD) method, we apply it to a cerebrovascular disease dataset of 20 cases CTP scanned at tube current $190 \mathrm{~mA}$ from our medical institute. Out of 20 subjects, 10 are used as training and validation (6 with CTP deficits in the brain and 4 normal), and the remaining 10 are used for testing purpose ( 5 with CTP deficits and 5 normal). For all 
experiments of SPD, the dictionary used are of size $64 \times 256$ designed to handle perfusion image patches of $8 \times 8$ pixels with 256 atoms in the dictionary. We download the online dictionary learning for sparse representation code from the authors' website 1 , and the model-based brain segmentation code 2 . The optimal parameters are obtained empirically from the training and validation dataset are: $\mu_{1}=0.01,0.02,0.04,0.08$ and $\mu_{2}=0.2,0.4,0.8,1$ for vessel, GM, WM and CSF. The threshold for vessel segmentation is $70 \mathrm{~mL} / 100 \mathrm{~g} / \mathrm{min}$ on the CBF map, as found to be the optimal value in our empirical experiments.

Since repetitive scanning of the same patient at different radiation doses is unethical, correlated Gaussian noise is added to the high-dose CTP data to simulate low-dose CTP data at $I \mathrm{~mA}$ following the practice in [12]: $I=\left(K^{2}\right.$. $\left.I_{0}\right) /\left(K^{2}+\sigma_{a}^{2} \cdot I_{0}\right)$, where $\sigma_{a}$ is the standard deviation of the added noise, $I_{0}=190 \mathrm{~mA}$ is the tube current at high-dose, $K=103.09 \mathrm{~mA}^{\frac{1}{2}}$ is a constant. Low tube current of $15.6 \mathrm{~mA}$ was simulated by adding correlated Gaussian noise with standard deviation of 25 . CBF maps computed from CTP data obtained at high tube current of $190 \mathrm{~mA}$ were regarded as the reference standard. We present both the visual and quantitative
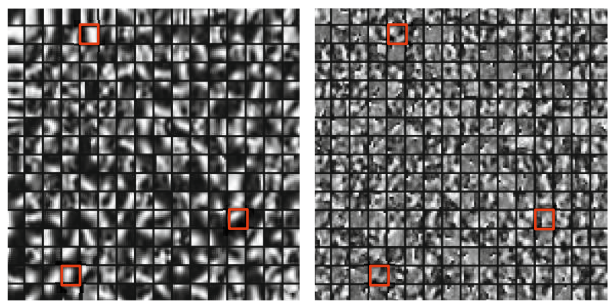

Fig. 2. Left: Global dictionaries learned using K-SVD. Right: Tissue-specific dictionary for white matter. The global dictionary is dominated by high-contrast, edge-like atoms, while the tissue-specific dictionary for WM has more lowcontrast, fine structured atoms, as highlighted by red boxes. results to demonstrate performance of the proposed method, with the comparison to cTSVD [13] and KSVD-SPD [3].

Tissue-Specific Dictionaries: Figure 2 shows the globally learned dictionary using KSVD and the tissue-specific dictionary for white matter. The global dictionary is trained on a dataset of $40,0008 \times 8$ patches of high-dose CBF perfusion maps randomly sampled from 10 training subjects and initialized with the redundant DCT dictionary. Each tissue-specific dictionary is trained using $10,0008 \times 8$ patches of the corresponding tissue category from the same training subjects. We could observe from the global dictionary that high-contrast patches with edges and corners dominate the dictionary atoms. In comparison, the tissue-specific dictionary for white matter preserves the texture and image characteristics for this tissue class.

CBF Perfusion Map: We then compare three methods by visually observing the estimated CBF perfusion maps of two patients. As shown in Figs. 3, among the three low-dose CBF maps, the CBF maps generated using our proposed TS-SPD algorithm recovers the information of high-dose CBF maps from the low-dose CTP data with best overall performance. The arteries and veins as well as the micro-vessels are better

\footnotetext{
${ }^{1}$ http: //spams-devel.gforge.inria.fr/

${ }^{2}$ http://sourceforge.net/projects/niftyseg/
} 


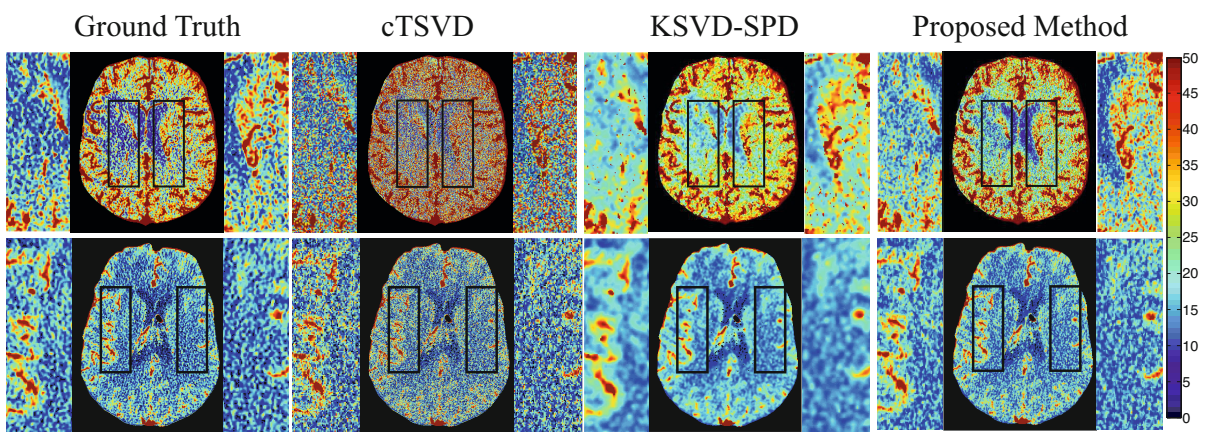

Fig. 3. CBF maps and zoomed-in regions. A 63-year-old female with acute stroke has an ischemic region in the right hemisphere of the brain (1st row) and a 35-year-old female with left middle cerebral artery (LMCA) perfusion deficit caused by aneurysmal SAH (2nd row). (Note representations in medical images display the sides of the images in reverse order) LMCA and RMCA are enlarged for comparison next to each image. The low-contrast tissue classes in the LMCA and RMCA regions are highly noisy in cTSVD images, and are over-smoothed by KSVD-SPD, while TS-SPD preserves the subtle variations and are closest to the ground truth.

Table 1. Quantitative comparison of PSNR $(\mathrm{dB})$ in CBF maps at low-dose are reported for 10 CTP cases by cTSVD, KSVD-SPD and our TS-SPD. Stroke and SAH indicate the two subjects in Fig. 3 respectively. The best performance is in bold-face type.

\begin{tabular}{c|ccc|ccc|ccc}
\hline PSNR & \multicolumn{3}{|c|}{ Brain } & \multicolumn{3}{|c|}{ GM } & \multicolumn{3}{c}{ WM } \\
& Stroke & SAH & All data & Stroke SAH & All data & Stroke & SAH & All data \\
\hline cTSVD & 43.51 & 34.87 & 33.57 & 12.81 & 15.94 & 15.91 & 19.99 & 18.65 & 17.82 \\
KSVD-SPD & 45.80 & 37.11 & 34.91 & 17.53 & 18.08 & 17.88 & 22.80 & 19.75 & 19.41 \\
TS-SPD & $\mathbf{4 7 . 8 4}$ & $\mathbf{3 8 . 3 8}$ & $\mathbf{3 6 . 6 5}$ & $\mathbf{1 8 . 9 2}$ & $\mathbf{1 9 . 6 6}$ & $\mathbf{1 9 . 9 1}$ & $\mathbf{2 5 . 0 2}$ & $\mathbf{2 2 . 5 6}$ & $\mathbf{2 2 . 2 8}$ \\
\hline
\end{tabular}

defined, while the delicate structures of the white matter and CSF are preserved. While the noise is greatly suppressed in the low-dose CBF maps for both enhancement algorithms, KSVD-SPD tends to smooth the image too much, especially the non-vessel structures. Our TS-SPD algorithm overcomes these drawbacks and preserves both the vessel boundaries and the low-contrast structures of WM and CSF with tissue-specific dictionaries and adaptive parameter settings for each tissue class.

Asymmetry: To visualize the asymmetry in the left and right middle cerebral artery in Fig. 3. we compute the intensity difference maps between LMCA and RMCA for three methods, as shown in Fig. 4. The intensity difference map of cTSVD is too noisy to identify the asymmetry of LMCA and RMCA vessel structures, while KSVD-SPD blurs the details of the vessel structure. The proposed method generates the different map with better contrast and spatial resolution for diagnosis of asymmetry in LMCA and RMCA.

Quantitative Comparisons: We report the PSNR (peak signal-to-noise-ratio) values for two cases in Fig. 3 and all testing subjects on the whole brain, GM and WM in Table 1. The GM and WM are the tissue regions in the brain affected by stroke and other 


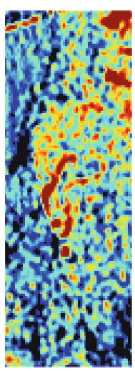

(a)

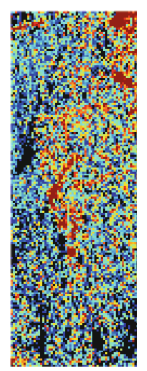

(b)

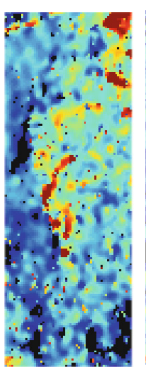

(c)

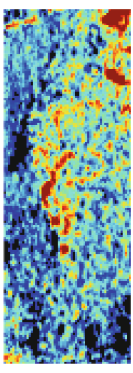

(d)

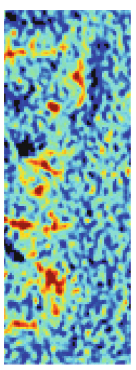

(a)

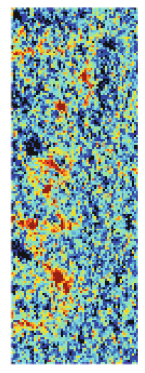

(b)

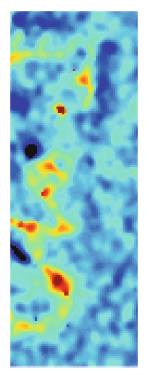

(c)

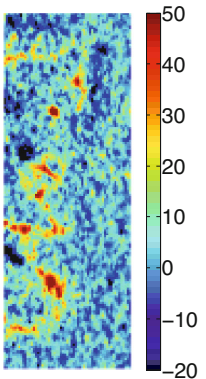

(d)

Fig. 4. Zoomed-in regions of the intensity difference maps between LMCA and RMCA of the acute stroke (left) and SAH (right) patients estimated by (a) Ground truth (b) cTSVD (c) KSVDSPD (d) Proposed TS-SPD. Arteries are delineated in red, CSF in blue.

ischemic processes. The proposed method again achieves the highest PSNR (usually $2 \sim 3 \mathrm{~dB}$ higher) in all cases, allowing for better discrimination ability of these brain regions, by preserving the tissue structures for differentiation of infarct core and ischemic penumbra in specific regions of the brain assisting neuroradiologists in diagnosis.

Ischemic Voxels Clustering: We also perform the clustering experiment as in [3] by aggregating all voxels (within VOI) from the normal hemisphere into a single "normal" cluster and the pathologic hemisphere into an "abnormal" cluster. To quantify the separability between normal and ischemic CBF values, we define the distance between these two clusters as: $d=\left(m_{1}-\right.$ $\left.m_{2}\right) / \sqrt{\sigma_{1}^{2} / n_{1}+\sigma_{2}^{2} / n_{2}}$, where $m_{1}, m_{2}$ are the means, and $\sigma_{1}$ and $\sigma_{2}$ are the standard deviations of $\mathrm{CBF}$ in the normal and ischemic clusters, $n_{1}$ and $n_{2}$ are the number of normal voxels ischemic voxels, respectively. We hypothesized that our TS-SPD algorithm to produce larger distance $d$, that is, to more definitely differentiate between normal and ischemic tissues. Table 2 shows the distance between normal and abnormal clusters for the two cases in Fig. 3 and all subjects with CTP deficits. TS-SPD separates the two clusters with greatest distance. One-tail paired t-test yields $p=0.051$ between cTSVD and KSVD-SPD, and $p=0.015$ between KSVD-SPD and TS-SPD.

\section{Conclusion}

In this paper, we have proposed a novel tissue-specific dictionary learning and deconvolution approach for CBF perfusion map enhancement in low-dose cerebral CTP. 
We take advantage of the distinctive image information of each tissue category available in the high-dose CBF maps to recover the missing texture and structural information in the low-dose CBF maps. This is achieved by performing a spatio-temporal sparse deconvolution based on tissue-specific dictionaries learned from high-dose CBF map segmentation. Our method consistently outperforms the state-of-art methods, especially in GM and WM where the cerebrovascular disease diagnoses mostly rely. In the future, we will evaluate the feasibility of applying our method in clinical practice using CTP acquired at low radiation dose in patients with cerebrovascular disease.

\section{References}

1. Miles, K.A., Griffiths, M.R.: Perfusion CT: a worthwhile enhancement? British Journal of Radiology 76(904), 220-231 (2003)

2. Wintermark, M., Lev, M.: Fda investigates the safety of brain perfusion CT. American Journal of Neuroradiology 31(1), 2-3 (2010)

3. Fang, R., Chen, T., Sanelli, P.C.: Sparsity-based deconvolution of low-dose perfusion CT using learned dictionaries. In: Ayache, N., Delingette, H., Golland, P., Mori, K. (eds.) MICCAI 2012, Part I. LNCS, vol. 7510, pp. 272-280. Springer, Heidelberg (2012)

4. Fang, R., Chen, T., Sanelli, P.C.: Towards robust deconvolution of low-dose perfusion CT: Sparse perfusion deconvolution using online dictionary learning. Medical Image Analysis (2013)

5. He, L., Orten, B., Do, S., Karl, W., Kambadakone, A., Sahani, D., Pien, H.: A spatio-temporal deconvolution method to improve perfusion CT quantification. IEEE Transactions on Medical Imaging 29(5), 1182-1191 (2010)

6. Calamante, F., Gadian, D., Connelly, A.: Quantification of bolus-tracking MRI: Improved characterization of the tissue residue function using Tikhonov regularization. Magnetic Resonance in Medicine 50(6), 1237-1247 (2003)

7. Yang, J., Wright, J., Huang, T., Ma, Y.: Image super-resolution as sparse representation of raw image patches. In: IEEE Conference on Computer Vision and Pattern Recognition, CVPR 2008, pp. 1-8. IEEE (2008)

8. Zhang, S., Zhan, Y., Metaxas, D.: Deformable segmentation via sparse representation and dictionary learning. Medical Image Analysis (2012)

9. Hoeffner, E., Case, I., Jain, R., Gujar, S., Shah, G., Deveikis, J., Carlos, R., Thompson, B., Harrigan, M., Mukherji, S.: Cerebral perfusion CT: Technique and clinical applications. Radiology 231(3), 632-644 (2004)

10. Van Leemput, K., Maes, F., Vandermeulen, D., Suetens, P.: Automated model-based tissue classification of MR images of the brain. IEEE Transactions on Medical Imaging 18(10), 897-908 (1999)

11. Mairal, J., Bach, F., Ponce, J., Sapiro, G.: Online dictionary learning for sparse coding. In: Proceedings of the 26th Annual International Conference on Machine Learning, pp. 689-696. ACM (2009)

12. Britten, A., Crotty, M., Kiremidjian, H., Grundy, A., Adam, E.: The addition of computer simulated noise to investigate radiation dose and image quality in images with spatial correlation of statistical noise: an example application to X-ray CT of the brain. British Journal of Radiology 77(916), 323-328 (2004)

13. Wittsack, H., Wohlschläger, A., Ritzl, E., Kleiser, R., Cohnen, M., Seitz, R., Mödder, U.: CT-perfusion imaging of the human brain: advanced deconvolution analysis using circulant singular value decomposition. Computerized Medical Imaging and Graphics 32(1), 67-77 (2008) 